"This is the pre-peer reviewed version of the following article: Dan Wen, Wei Liu, Anne-Kristin Herrmann, Danny Haubold, Matthias Holzschuh, Frank Simon, and Alexander Eychmüller (2016). Simple and Sensitive Colorimetric Detection of Dopamine Based on Assembly of Cyclodextrin-Modified Au Nanoparticles. Small. Volume 12, Issue 18, May 11, 2016, Pages 2439-2442, which has been published in final form at DOI: 10.1002/smll.201503874.

This article may be used for non-commercial purposes in accordance with Wiley Terms and Conditions for Self-Archiving."

\title{
Simple and Sensitive Colorimetric Detection of Dopamine Based on Assembly of Cyclodextrin-Modified Au Nanoparticles
}

Dan Wen, Wei Liu, Anne-Kristin Herrmann, Danny Haubold, Matthias Holzschuh, Frank Simon, and Alexander Eychmüller*

[*] Dr. D. Wen, Dr. W. Liu, Dr. A.-K. Herrmann, D. Haubold, Prof. A. Eychmüller

Physical Chemistry, TU Dresden

Bergstrasse 66b, 01062 Dresden (Germany)

E-mail: alexander.eychmueller@ chemie.tu-dresden.de

M. Holzschuh, Dr. F. Simon

Physical Chemistry and Physics of Polymers

Leibniz Institute of Polymer Research Dresden

Hohe Strasse 6, 01069 Dresden (Germany)

[**] This work is supported by DFG GRK1401, the DFG project EY16/10-2, and the AEROCAT project (ERC-2013-ADG 340419). D. W. acknowledges the support from the Alexander von Humboldt Foundation.

\section{Keywords}

Dopamine, colorimetric detection, Au nanoparticles, assembly, beta-cyclodextrin 


\section{Introduction}

Dopamine is one of the most important neurotransmitters of both the central and peripheral nervous systems and disorder of its concentration has been associated with a series of neural diseases, such as Parkinson's and Alzheimer's diseases.[1] Hence, the development of a simple method to detect dopamine is of significant importance, at best at low cost and high sensitivity. A great number of elegant methods, including electrochemical strategies, chemiluminescence, chromatography, capillary electrophoresis, and spectroscopic approaches have been successfully applied in the dopamine determination.[2] However, most of them require expensive equipment and/or complicated operations. The colorimetric assay shows great promise to solve the concerned issues, with theoretical and technical simplicity.[3] In the past decades, much research has centered on the colloidal nanoparticles (NPs) that become the most important building blocks for the fabrication of sensitive optical nanosensors.[4] Specifically the utilization of Au NPs as colorimetric sensing platforms is gaining considerable attention due to the high extinction coefficients and the unique size dependent optical property, which opens the access to high sensitivity with unsophisticated transducers.[5] A conventional strategy for dopamine detection with Au NPs is the rational design of their surface chemistry, which trigger the dis-/assembly of the particles and the accompanying color changes upon recognition by the target molecule.[6] Although the benefits of these sensors are already apparent, most of the Au NP optical probes involve quite complicated procedures on ligands, such as pre-functionalization or post-exchange, and sometimes external metal salts or ions are needed in the sensing system.[7]

In this work, we demonstrate a facile colorimetric strategy for the determination of dopamine, where the natural beta-cyclodextrin modified Au nanoparticles ( $\beta$-CD-Au NPs) were directly employed as optical probes. The method relies on a controlled destabilization of the $\beta-\mathrm{CD}-\mathrm{Au}$ NPs and growth of the Au assemblies (i.e., "peanut-shaped", short chain, and wire-network nanostructures) by means of simply tuning the dopamine concentration, as illustrated in Figure 1C. With the color change in the assembly process, the amount of the target is quantified. It is noted that there is no demand for general pretreatments like surface ligand exchange/prefunctionalization for the NPs or additional agents during the determination, which enables a simple and sensitive detection of dopamine with a detection limit of $20 \mathrm{nM}$. Additionally, the 
optical sensor exhibits high selectivity for the co-existing interferences as well as the analogues, carrying promise for a real sample test.

\section{Results and discussion}

\section{Synthesis and characterization of the colorimetric probes}

The Au NP-based colorimetric probes, decorated by $\beta-\mathrm{CD}$, were directly synthesized by reducing chloroauric acid with sodium borohydride in the presence of natural $\beta-\mathrm{CD}$. The resulting wine-red Au colloid shows the characteristic surface plasmon resonance (SPR) peak at $513 \mathrm{~nm}$, indicating the formation of Au NPs with a diameter of several nanometers, as demonstrated in curve c, Figure S1A, Supporting Information. To further confirm that the band is indeed caused by $\mathrm{Au}$ NPs, the colloidal dispersion was analyzed by transmission electron microscopy (TEM). Spherical NPs with an average diameter of $4.9 \pm 1.0 \mathrm{~nm}$ were observed (Figure S1B-C). Furthermore, high-resolution TEM images indicate that the $\beta$-CD-Au NPs display well-defined lattice planes with interplanar $d$ spacings of $0.23 \mathrm{~nm}$, corresponding to the (111) planes of face-centered cubic Au (Figure S1D). $\beta$-CD is expected to be adsorbed on the surface of the Au NPs playing a role as stabilizing agent, which is further confirmed by the following characterizations. Fourier transform infrared (FT-IR) spectra in Figure S2 show that the $\beta$-CD-Au NPs reveal the characteristic absorption peaks of $\beta-C D$, suggesting that the Au NP surface is indeed capped with $\beta$-CD molecules. X-ray photoelectron spectroscopy (XPS) (Figure S3) was performed to gain more insight into the structure of the as-prepared $\beta$-CD-Au NPs. The C $1 \mathrm{~s}$ spectrum depicts the presence of a large number of functionalized carbon-containing molecules, which also confirms the existence of $\beta-C D$ in the $\beta-C D-A u$ NPs.[8] Additionally, the recorded $\mathrm{Au} 4 \mathrm{f}$ spectrum is composed of $\mathrm{Au} 4 \mathrm{f} 7 / 2$ and $\mathrm{Au} 4 \mathrm{f} 5 / 2$ peaks with binding energies at 84.0 and $87.8 \mathrm{eV}$, respectively. These are typical values for metallic gold which implies the complete reduction of the Au precursors during the direct synthesis of the $\beta-\mathrm{CD}-\mathrm{Au}$ NPs.

\section{Assembly of the $\beta-C D-A u$ NPs}

The initial Au colloid was wine-red and stable in the as-prepared state (Figure 1A, a), while the introduction of dopamine stimulated the assembly of the $\beta$-CD-Au NPs. Upon the addition of different amounts of dopamine, except case b, the color of the Au NP solutions became darker (c), purplish red (d, e), bluish violet (f), and even gray (g), as depicted in Figure 1A. These color 
changes underline the structural change of the Au NPs, which was further examined by UV-Vis spectroscopy and verified by TEM. As shown in Figure 1B, the SPR peaks of the samples b-g are broadened with a slight red shift from 513 to $522 \mathrm{~nm}$ accompanied by an increase of the long-wavelength SPR band. The latter is indicative for the controlled growth of Au assemblies. One may notice that high concentrations of dopamine result in a decrease of the intensity of the SPR bands because of some Au precipitation (curve g).
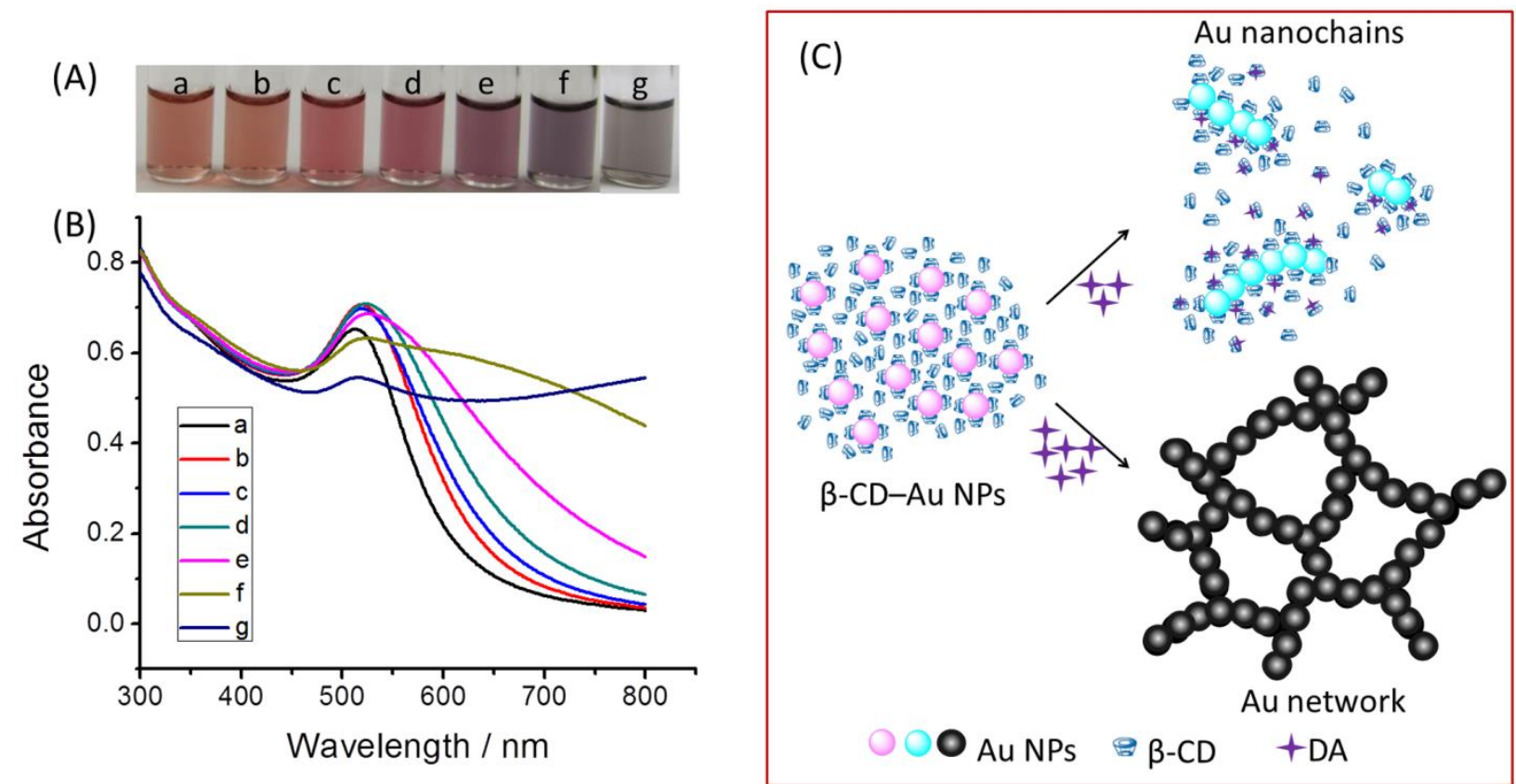

Figure 1. (A) Photographs of the $\beta-\mathrm{CD}-\mathrm{Au}$ NP colloids before (a) and after the addition of dopamine (b-g) and their corresponding UV-Vis spectra (B). The concentrations of dopamine are 2.0, 2.1, 2.25, 2.75, 3.6 and 7.5 $\mu \mathrm{M}$, respectively. (C) Schematic representation of the dopamineinduced Au NP assembly.

TEM analysis was conducted to ascertain the assembly states of the NPs mediated by dopamine, which are in line with the photographs and the absorption spectra. The as-prepared $\beta$ CD-Au NPs were well-dispersed, even though a few "peanut-shaped" nanostructures were observed (Figure S1B) which could be facilitated by the weak protection of the Au NPs with natural CD.[9] Figure 2 and S4 display representative Au nanoarchitectures induced by the addition of $2.0,2.25,3.6$ and $7.5 \mu \mathrm{M}$ of dopamine. It is interesting to find that with the addition of low concentrations more "peanut-shaped" nanostructures become apparent (Figure 2A), although no obvious color changes in the Au solution have been observed at this stage. The Au 
nanochains grow longer and form branching points with the dopamine concentration increasing to 2.25 and $3.6 \mu \mathrm{M}$ (Figure $2 \mathrm{~B}-\mathrm{C}$ ). By further increasing the concentration and the interaction time, three-dimensional (3D) network-like Au nanowires are formed, being highly branched and revealing an average diameter of $5.7 \pm 1.1 \mathrm{~nm}$ which is similar to the size of the original NPs (Figure 2D). It is of particular significance that the Au NP assembly is not the result of simple aggregation into larger particles but occurring in a controlled manner, unlike many other approaches.[10] This dopamine mediated Au NP assembly is probably due to dual interactions of the host-guest complexation between dopamine and $\beta-\mathrm{CD}[11]$, and the specific affinity between dopamine and the Au cores[12].

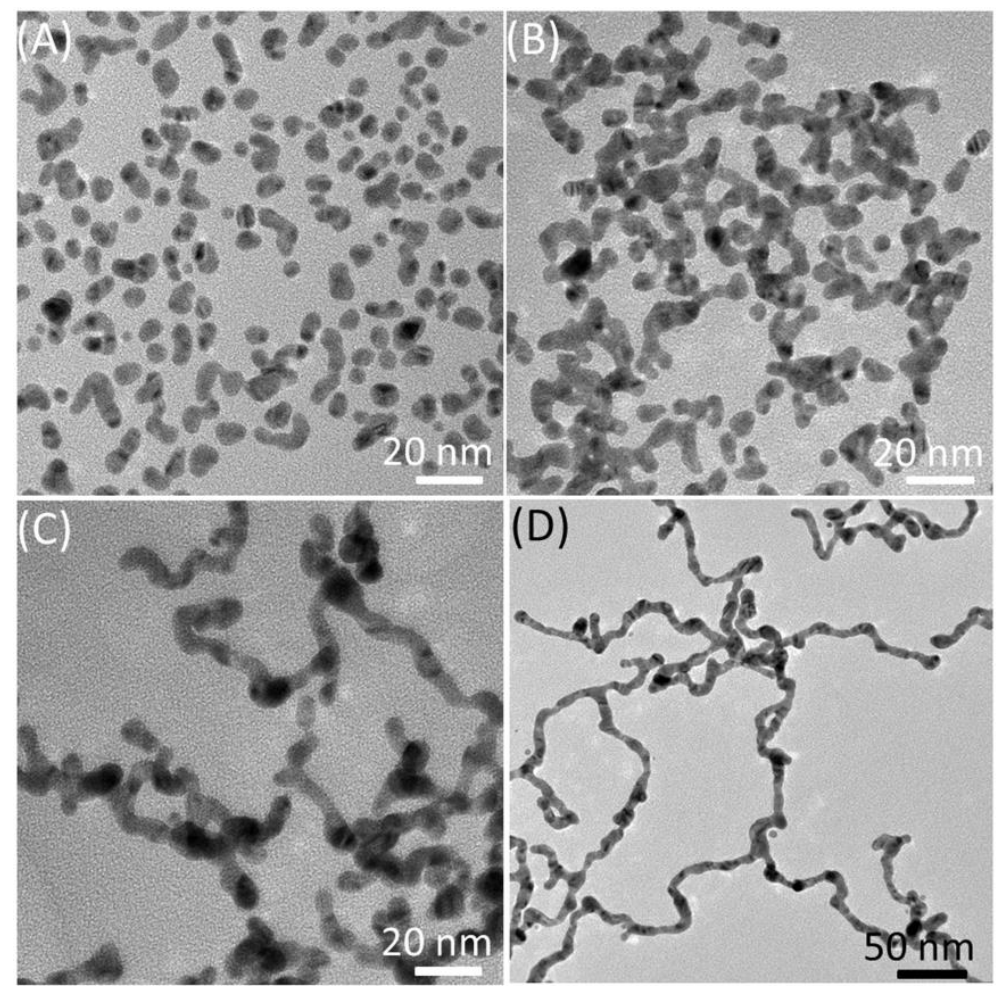

Figure 2. TEM images of $\mathrm{Au}$ assemblies upon the addition of different concentrations of dopamine: (A) 2.0, (B) 2.25, (C) 3.6 and (D) $7.5 \mu \mathrm{M}$.

\section{Colorimetric determination of dopamine}

Inspired by the concentration-dependent assembly triggered by dopamine, the $\beta$-CD-Au NPs are anticipated as optical probes for a facile colorimetric determination. The Au NP assembly was reflected in the UV-Vis spectrum, with a decrease in absorption at about $520 \mathrm{~nm}$ and an 
increase of the long-wavelength SPR band. Here the ratio of the absorption at 520 and $650 \mathrm{~nm}$ $\left(\mathrm{A}_{650} / \mathrm{A}_{520}\right)$ was also used for a quantitative analysis of dopamine, which below $2 \mu \mathrm{M}$ increases only slightly with an increasing concentration, while becomes significant in the concentration range above $2 \mu \mathrm{M}$ (Figure S5). A probable reason for this observation could be the existence of free $\beta$-CD in $\mathrm{Au}$ colloids due to its excess amount during the synthesis, Figure S2A and Supporting Information. Thus the sensitivity for detection can be greatly improved by pre-adding a certain amount of dopamine (i.e., $2 \mu \mathrm{M}$ ) to the Au NP sample.[6b,13]

With the addition of $2 \mu \mathrm{M}$ of dopamine in the $\beta$-CD-Au NPs solution in advance, the kinetic investigations on different additional analyte concentrations (i.e., 0, 40, 100 and $750 \mathrm{nM}$ ) were performed, as shown in Figure $3 \mathrm{~A}$ and $\mathrm{S} 6$. The results suggest that the ratio $\mathrm{A}_{650} / \mathrm{A}_{520}$ first increases rapidly and then remains nearly constant after about $30 \mathrm{~min}$. As a compromise between response time and sensitivity, $30 \mathrm{~min}$ were selected as the incubation time for the further detection. Under thus optimized conditions, a good linear relationship is found between the ratio $\mathrm{A}_{650} / \mathrm{A}_{520}$ and the concentration of the further added dopamine over the range of $20-250 \mathrm{nM}$ $\left(\mathrm{R}^{2}=0.997\right)$ and $350-1600 \mathrm{nM}\left(\mathrm{R}^{2}=0.990\right)$, respectively (Figure 3B and C). The limit of detection based on a signal/noise ratio of 3 was estimated to be as low as $3 \mathrm{nM}$. This is more sensitive than those of colorimetric detection based on other types of Au NP probes.[7b,14] It is reported that the basal level of dopamine in the extracellular fluid of the caudate nucleus is $0.01-1 \mu \mathrm{M}$ for a healthy individual.[15] Therefore, the present strategy in this study is promising to detect dopamine in body fluids. In addition, the selectivity of the optimized sensor for dopamine was evaluated by monitoring the extinction ratio $\left(\mathrm{A}_{650} / \mathrm{A}_{520}\right)$ in the presence of some interferences possible co-existing in the body fluid (Figure 3D and S7). The existence of $\mathrm{H}_{2} \mathrm{O}_{2}$, glucose, lactic acid, ascorbic acid, uric acid, and some analogues like pyrocatechol, 2-phenylethylamine, epinephrine and norepinephrine shows negligible or only low responses at 20 fold-concentrations, compared with dopamine. Moreover, the stability of the $\beta-\mathrm{CD}-\mathrm{Au}$ NPs based optical probes remains nearly constant under $4{ }^{\circ} \mathrm{C}$ in the refrigerator, as displayed in Figure S8. 

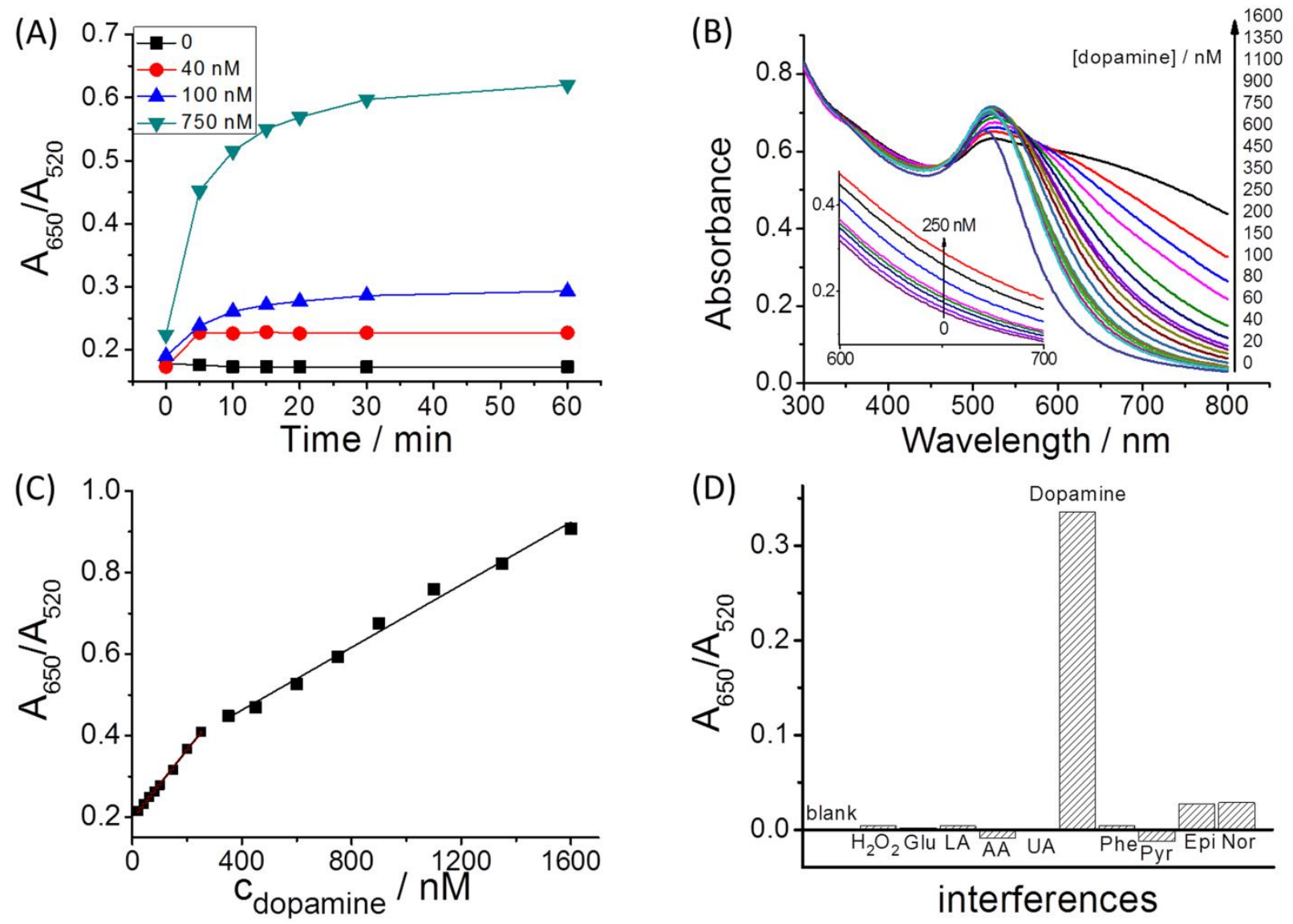

Figure 3. (A) Plot of $A_{650} / A_{520}$ versus time with additional dopamine concentration at $0,40,100$ and $750 \mathrm{nM}$, (B) UV-Vis spectra of the optimized $\beta-\mathrm{CD}-\mathrm{Au}$ NPs probe with the addition of dopamine at different concentrations. (C) Linear plots of $\mathrm{A}_{650} / \mathrm{A}_{520}$ versus dopamine concentration, (D) Selectivity investigation for the optimized probe over different physiological interferences: Glu (glucose); LA (lactic acid); AA (ascorbic acid); UA (uric acid); Phe (2phenylethylamine); Pyr (pyrocatechol); Epi ((-)-Epinephrine); Nor ((-)-Norepinephrine). [dopamine] $=0.5 \mu \mathrm{M}$ and [interferences] $=10 \mu \mathrm{M}$.

Our $\beta$-CD-Au NP-based colorimetric probes exhibit excellent performance towards the dopamine detection, such as high sensitivity, selectivity and stability, as well as simplicity in the sensor fabrication. Furthermore, the relative standard deviation (RSD) for eleven repeated measurements of $50 \mathrm{nM}$ dopamine is $1.0 \%$, illustrating that the response of the optimized $\mathrm{Au}$ NP-probe is highly reproducible. To demonstrate the viability of this Au NPs probe for colorimetric analysis of actual samples, we carried out the measurement of dopamine in artificial cerebrospinal fluid (a-CSF). The recovery was investigated by adding a known amount of 
analyte. The results summarized in Table S1 indicate that the recovery $(95.8 \sim 103.1 \%)$ and precision (RSD: $1.9 \sim 3.1 \%, \mathrm{n}=7$ ) of the proposed method applied to determine dopamine in aCSF are satisfactory.

\section{Conclusion}

To summarize, natural $\beta$-CD modified Au NPs have been first demonstrated as optical probes for the determination of dopamine. Upon the addition of the target, $\beta-\mathrm{CD}-\mathrm{Au}$ NPs readily assemble into "peanut-shaped", short nanochain, and inter-fused 3D nanowire-network architectures. With the color change occurring when dopamine was added successively to a $\mathrm{Au}$ NP solution, a sensitive and selective colorimetric method for dopamine detection is successfully developed. The presented strategy has low technical and instrumental demands, and is further simplified by neither needing ligand exchange nor cross-linkers in the sensing process. Such results could not only provide a methodology to design AuNP-based colorimetric assays with high specificity and sensitivity, but also hold promise of fine $\mathrm{Au}$ assemblies for further applications which are currently underway in our laboratory. 


\section{Experimental Section}

Synthesis of $\beta-C D-A u$ NPs: The $\beta-C D$ modified Au NPs were synthesized according to the previously reported method with modifications.[16] $174 \mu \mathrm{L}$ of $10 \% \mathrm{HAuCl}_{4}$ was added into 199 $\mathrm{mL} 10 \mathrm{mM} \beta-\mathrm{CD}$ under continuously stirring for $10 \mathrm{~min}$, and then $1 \mathrm{~mL}$ of a freshly prepared $180 \mathrm{mM} \mathrm{NaBH} 4$ aqueous solution was quickly added into the solution. The resulting solution was kept stirring for about $2 \mathrm{~h}$ to get wine red Au colloids.

Dopamine-induced assembly and its colorimetric determination: Different concentrations of dopamine with the same volume $(4 \mu \mathrm{L})$ were added into $2 \mathrm{~mL}$ of the $\beta$-CD-modified Au NPs with slightly shaking for $30 \mathrm{~min}$ and the above solution was recorded by UV-Vis spectroscopy. For the colorimetric detection of dopamine, $2 \mu \mathrm{M}$ of dopamine was added in advance for optimization, and then $2 \mathrm{~mL}$ of the $\beta-\mathrm{CD}-\mathrm{Au}$ NPs were mixed with $4 \mu \mathrm{L}$ of different concentrations of dopamine. The a-CSF sample composed of $150 \mathrm{mM} \mathrm{NaCl}, 3.0 \mathrm{mM} \mathrm{KCl}, 1.4$ $\mathrm{mM} \mathrm{CaCl}_{2}, 0.8 \mathrm{mM} \mathrm{MgCl}_{2}$, and $1.0 \mathrm{mM}$ sodium phosphate was home-made.

Apparatus and Measurements: A diluted drop of each sample after standing quietly for $24 \mathrm{~h}$ was evaporated on a carbon coated copper grid and analyzed by transmission electron microscopy (TEM) on a Zeiss Libra 200 and TECNAI T20 at an accelerating voltage of $200 \mathrm{kV}$, while the preparation time for the $\mathrm{Au}$ assemblies sample induced by $7.5 \mu \mathrm{M}$ dopamine was 1 week. After 30 minutes' interaction with dopamine and Au NPs, UV-Vis spectroscopy was recorded on a Cary 50 UV-Vis Spectrophotometer. Fourier transform infrared (FT-IR) spectroscopy was performed on a Thermo Scientific Nicolet 8700 FT-IR Spectrometer configured with a Smart iTR diamond accessory. X-ray photoelectron spectroscopy (XPS) studies were carried out with an AXIS ULTRA photoelectron spectrometer (KRATOS ANALYTICAL, Manchester, England). Zeta-Potential measurements were performed on a DelsaNano C, particle analyzer (Beckman Coulter Corporation). 


\section{References}

[1] a) I. Kopin, Pharmacol. Rev. 1985, 37, 333; b) T. Lee, L. X. Cai, V. S. Lelyveld, A. Hai, A.

Jasanoff, Science 2014, 344, 533; b) M. N. Zhang, P. Yu, L. Q. Mao, Acc. Chem. Res. 2012, 45, 533.

[2] a) P. E. Phillips, G. D. Stuber, M. L. Heien, R. M. Wightman and R. M. Carelli, Nature 2003, 422, 573; b) S. F. Liu, X. Zhang, Y. M. Yu, G. Z. Zou, Anal. Chem. 2014, 86, 2784; c) À. Dago, J. Navarro, C. Ariño, , J. M. Díaz-Cruz, M. Esteban, J. Chromatogr. A, 2015, 1409, 210; d) M. J. Schöning, M. Jacobs, A. Muckc, D.-T. Knobbe, J. Wang, M. Chatrathi, S. Spillmann, Sens. Actuators B Chem. 2005, 108, 688; e) J. S. Lee, J. Oh, S. G. Kim, J. Jang, 2015, 11, 2399; f) J. P. Yuan, D. Wen, N. Gaponik, A. Eychmüller, Angew. Chem. Int. Ed. 2013, 52, 976.

[3] a) J. B. Zhu, T. Li, L. Zhang, S. Dong, E. G. Wang, Biomaterials 2011, 32, 7318; b) W. Zhou, X. Gao, D. B. Liu, X. Y. Chen, Chem. Rev. 2015, 115, 10575.

[4] a) P. D. Howes, R. Chandrawati, M. M. Stevens, Science 2014, 346, 6205; b) L. H. Guo, Y. Xu, A. R. Ferhan, G. N. Chen, D.-H. Kim, J. Am. Chem. Soc. 2013, 135, 12338; c) S. Mao, J. B. Chang, G. H. Zhou, J. H. Chen, Small 2015, 11, 5336.

[5] a) F. A. Wang, X. Q. Liu, C. H. Lu, I. Willner, ACS Nano 2013, 7, 7278; b) R. Rica, A. H. Velders, Small 2011, 7, 66; c) J. J. Deng, P. Yu, Y. X. Wang, L. F. Yang, L. Q. Mao, Adv. Mater. 2014, 26, 6933; d) X. Zhang, C. A. Marocico, M. Lunz, V. A. Gerard, Y. K. Gun'ko, V. Lesnyak, N. Gaponik, A. S. Susha, A. L. Rogach and A. L. Bradley, ACS Nano 2012, 6, 9283.

[6] a) C. M. Yu, M. Luo, F. Zeng, F. Y. Zheng, S. Z. Wu, Chem. Commun. 2011, 47, 9086; b) B. Kong, A. W. Zhu, Y. P. Luo, Y. Tian, Y. Y. Yu, G. Y., Angew. Chem. Int. Ed. 2011, 50, 1837. [7] a) X. Chen, S. G. Parker, G. Zou, W. Su, Q. J. Zhang, ACS Nano 2010, 4, 6387; b) L. Liu, S. J. Li, L. L. Liu, D. H. Deng, N. Xia, Analyst 2012, 137, 3794; c) N. Yusoff, A. Pandikumar, R. Ramaraj, H. N. Lim, N. M. Huang, Microchim. Acta 2015, 182, 2091.

[8] Y. J. Guo, S. J. Guo, J. T. Ren, Y. M. Zhai, S. J. Dong, E. K. Wang, ACS Nano 2010, 4, 4001. [9] a) T. Huang, F. Meng, L. M. Qi, J. Phys. Chem. C 2009, 113, 13636; b) W. Liu, A.-K. Herrmann, D. Geiger, L. Borchardt, F. Simon, S. Kaskel, N. Gaponik, A. Eychmüller, Angew. Chem. Int. Ed. 2012, 51, 5743.

[10] a) J. Liu, J. Alvarez, W. Ong, A. E. Kaifer, Nano Lett. 2001, 1, 57; b) H. Li, D. X. Chen, Y. L. Sun, Y. B. Zheng, L. L. Tan, P. S. Weiss, Y. W. Yang, J. Am. Chem. Soc. 2013, 135, 1570. 
[11] a) R. Djemil, D. Khatmi, J. Comput. Theor. Nanosci. 2012, 9, 1571; b) G. Alarcon Angeles, B. Pérez López, M. Palomar-Pardave, M. T. Ramírez-Silva, S. Alegret, A. Merkoc, Carbon 2008, 46, 898-906.

[12] a) A. C. Templeton, M. J. Hostetler, C. T. Kraft, R. W. Murray, J. Am. Chem. Soc. 1998, 120, 1906; b) H. C. Lee, T. H. Chen, W. L. Tseng, C. H. Lin, Analyst 2012, 137, 5352.

[13] K. L. Ai, y. L. Liu, 1. H. Lu, J. Am. Chem. Soc. 2009, 131, 9496.

[14] a) Y. F. Zhang, B. X. Li, X. L. Chen, Microchim Acta 2010, 168, 107; b) J. J. Feng, H. Guo, Y. F. Li, Y. H. Wang, W. Y. Chen, A. J. Wang, ACS Appl. Mater. Interfaces 2013, 5, 1226; c) S. Palanisamy, X. H. Zhang, T. He, J. Mater. Chem. B 2015, 3, 6019.

[15] a) J. B. Justice, J. Neurosci. Methods 1993, 48, 263; b) C. S. Chaurasia, C. E. Chen, C. R. Ashby, Jr., J. Pharm. Biomed. Anal. 1999, 19, 413.

[16] Y. L. Liu, K. B. Male, P. Bouvrette, J. H. T. Luong, Chem. Mater. 2003, 15, 4172. 


\section{Table of Contents}

A controlled assembly of natural beta-cyclodextrin modified Au NPs mediated by dopamine was demonstrated. Furthermore, we established a simple and sensitive colorimetric detection for dopamine by the concentration-dependent assembly.

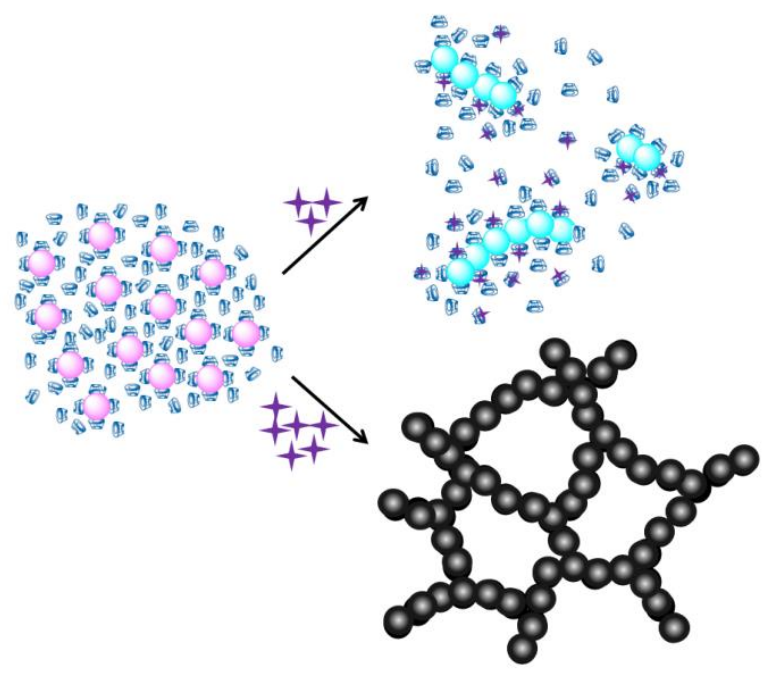

